Editorial

\title{
Neuroinflammation Update: New Insights and Future Directions
}

\author{
Kevin Rostasy ${ }^{1}$ \\ ${ }^{1}$ Division of Pediatric Neurology, Department of Pediatrics I, Innsbruck \\ Medical University, Innsbruck, Austria \\ Neuropediatrics 2013;44:295-296.
}

The recent discovery that mutations in the innate immune response that predispose children to Herpes simplex virus encephalitis or autoantibody-mediated diseases (e.g., $N$-methyl D-aspartate encephalitis) have deepened our knowledge about the diverse spectrum of acquired inflammatory diseases of the central nervous system (CNS). ${ }^{1,2}$ The detection of immunoglobulin $\mathrm{G}(\mathrm{IgG})$ antibodies against aquaporin-4 (AQP4) in subjects with neuromyelitis optica (NMO) and the subsequent proof that these autoantibodies are instrumental in the disease process have further drawn attention to B-cell-mediated mechanisms in acquired inflammatory demyelinating diseases, such as, multiple sclerosis (MS) and acute disseminated encephalomyelitis (ADEM). ${ }^{3,4}$

This emerging field of pediatric neurology that shares common ground with other pediatric specialties, including infectious diseases, rheumatology, oncology, and psychiatry, was one of the main topics at the 39th Annual Meeting of the Society of Neuropediatrics at Innsbruck, Austria.

For this special edition of Neuropediatrics entitled "Neuroinflammation Update: New Insights and Future Directions," we asked the speakers of plenary sessions with a neuroimmunological focus to provide an overview of the new developments and their consecutive clinical relevance based on their presentations at the 39th Annual Meeting of the Society of Neuropediatrics at Innsbruck, Austria.

The topics chosen are diverse and reflect the different areas of pediatric neurology in which neuroinflammation plays an important primary or secondary role in the disease process. The first contribution is by Rostasy and Reindl that addresses the role of autoantibodies in inflammatory demyelinating diseases of the CNS with a particular focus on antimyelin oligodendrocyte glycoprotein-IgG antibodies, which are present in a subgroup of patients with ADEM, AQP4-IgG seronegative $\mathrm{NMO}$, and recurrent optic neuritis. ${ }^{5}$ In the second article, Tardieu and Deiva describe the mimics of childhood MS, providing important clues for the reader on how to separate the different diseases. ${ }^{6}$ The third contribution is by Kornek who gives a detailed overview of the current treatment regimens for children and the emerging new oral medications for adult patients with MS, which will have major implications for children with MS in the future. ${ }^{7}$ Muller-Felber and colleagues provide an extensive review of pediatric idiopathic inflammatory myopathies with an update on diagnostic and treatment strategies. ${ }^{8}$ Hero and Schleiermacher report what is known about opsoclonusmyoclonus syndrome (OMS). They further introduce the OMS-European treatment trial to a wider audience that has started recruiting children this year and underscores the fact that international collaboration is mandatory to improve the care of children with rare neurological diseases. ${ }^{9}$ Ravizza et al contributed an excellent article on the importance of immune activation in the brain and its possible pathogenetic role in the propagation and maintenance of seizures. ${ }^{10}$ The next invited article by Mohammad et al gives an update on the role of autoantibodies in children with movement disorders. ${ }^{11}$

The next article is by Zotter et al who show that children after an episode of Lyme neuroborreliosis (LNB) have a normal neuropsychological profile in contrast to their adult counterparts who report symptoms, including memory problems and attention deficits. ${ }^{12}$

Last but not least, we have a book review by Boltshauser for the benefit of the readers. ${ }^{13}$ We hope this compilation meets the expectations of the readers of Neuropediatrics and proves to be informative and interesting.

\section{References}

1 Casrouge A, Zhang SY, Eidenschenk C, et al. Herpes simplex virus encephalitis in human UNC-93B deficiency. Science 2006; 314(5797):308-312

2 Dalmau J, Gleichman AJ, Hughes EG, et al. Anti-NMDA-receptor encephalitis: case series and analysis of the effects of antibodies. Lancet Neurol 2008;7(12):1091-1098

3 Papadopoulos MC, Verkman AS. Aquaporin 4 and neuromyelitis optica. Lancet Neurol 2012;11(6):535-544 (c) 2013 Georg Thieme Verlag KG Stuttgart · New York
DOI http://dx.doi.org/ 10.1055/s-0033-1360669. ISSN 0174-304X.
Address for correspondence Kevin Rostasy, MD, Division of

Pediatric Neurology, Department

of Pediatrics I, Medical University

Innsbruck, Anichstrasse 35,

A-6020 Innsbruck, Austria

(e-mail: kevin.rostasy@uki.at).
Issue Theme Neuroinflammation Update: New Insights and Future Directions; Guest Editor, Kevin Rostasy, MD. 
4 Meinl E, Krumbholz M, Hohlfeld R. B lineage cells in the inflammatory central nervous system environment: migration, maintenance, local antibody production, and therapeutic modulation. Ann Neurol 2006;59(6):880-892

5 Rostasy K, Reindl M. Role of autoantibodies in acquired inflammatory demyelinating diseases of the central nervous system in children. Neuropediatrics 2013;44(6):297-301

6 Tardieu M, Deiva K. Rare inflammatory diseases of the white matter and mimics of multiple sclerosis and related disorders. Neuropediatrics 2013;44(6):302-308

7 Kornek B. Treatment of pediatric multiple sclerosis. Neuropediatrics 2013;44(6):309-313

8 Muller-Felber W, Wanschitz J, Vill K, Baumann M. Pediatric idiopathic inflammatory myopathies: an update on diagnostic and treatment strategies. Neuropediatrics 2013;44(6): 314-323

9 Hero B, Schleiermacher G. Update on pediatric opsoclonus myoclonus syndrome. Neuropediatrics 2013;44(6):324-329

10 Ravizza T, Kostoula C, Vezzani A. Immunity activation in brain cells in epilepsy: mechanistic insights and pathological consequences. Neuropediatrics 2013;44(6):330-335

11 Mohammad SS, Ramanathan S, Brilot F, Dale RC. Autoantibody-associated movement disorders. Neuropediatrics 2013;44(6):336-345

12 Zotter S, Koch J, Schlachter K, et al. Neuropsychological profile of children after an episode of neuroborreliosis. Neuropediatrics 2013;44(6):346-353

13 Boltshauser E. Disorders of the spinal cord in children [Book Review]. Neuropediatrics 2013;44(6):354 